\title{
Conway irreducible hyperbolic knots with two common covers
}

\author{
Luisa Paoluzzi
}

\begin{abstract}
For each pair of coprime integers $n>m \geq 2$ we construct pairs of non equivalent Conway irreducible hyperbolic knots with the same $n$-fold and $m$-fold cyclic branched covers.
\end{abstract}

Mathematics Subject Classification (2000). Primary 57M25; Secondary 57M12, 57M50.

Keywords. Hyperbolic knots; cyclic branched covers; orbifolds.

\section{Introduction}

This paper is devoted to the problem of understanding whether hyperbolic knots are determined by any two of their cyclic branched covers. It is shown in [16, Theorem 3] that hyperbolic knots are determined by their $m$-fold and $n$-fold cyclic branched covers, for all integers $n>m>2$ which are not coprime, and that this remains true also for $m=2$ assuming in addition that the knots are $\pi$-hyperbolic or, more generally, Conway irreducible (see [8, proof of Proposition 1]).

On the other hand, for any fixed pair of coprime integers $n>m>2$, examples of inequivalent hyperbolic knots with the same $n$-fold and $m$-fold cyclic branched covers are given in [15], and for $m=2$ and arbitrary $n>2$ in [9]; all examples constructed in [15] and [9] are Conway reducible, so the above problem remained open for the most basic class of Conway irreducible hyperbolic knots (which are exactly the hyperbolic knots whose 2-fold cyclic branched cover admits no decomposition along spheres and contains no incompressible tori), and for the subclass of $\pi$-hyperbolic knots.

The first main result of the present paper is the following theorem which presents the first examples of inequivalent Conway irreducible hyperbolic knots which share two common cyclic branched covers.

Theorem 1. Let $n, m \geq 2$ be two coprime integers. There exists a pair of nonequivalent Conway irreducible hyperbolic knots with the same $n$-fold and $m$-fold cyclic branched covers. 
More precisely, we shall construct non-equivalent $\pi$-hyperbolic knots with two common cyclic branched covers.

It was claimed in [8] that Conway irreducible hyperbolic knots are determined by their 2-fold and $n$-fold cyclic branched covers, for any $n>2$, but the proof contains a gap (see the Erratum [11]) and Theorem 1 shows that this is in fact not true. However it remains true under some additional hypothesis:

Theorem 2. Let $K$ and $K^{\prime}$ be two $\pi$-hyperbolic knots, and let $n>2$ be an integer. Assume that $K$ admits a unique Seifert surface up to isotopy. If $K$ and $K^{\prime}$ have the same 2 -fold and $n$-fold cyclic branched covers, then $K$ and $K^{\prime}$ are equivalent.

To prove Theorem 1, we apply the method of construction used in [15], and we shall show that this is the only possible way to obtain such examples (see Proposition 2).

Zimmermann's method consists in showing that to obtain pairs of inequivalent hyperbolic knots with two common cyclic branched covers it suffices to construct a three-component link with certain additional properties, namely a hyperbolic link with prescribed symmetries. The three-component links considered by Zimmermann in [15] are Conway reducible, and his choice is motivated by the fact that for these links it is not difficult to check whether they are hyperbolic, to compute their symmetries and to find infinitely many different examples. However, these links correspond to pairs of non-equivalent hyperbolic knots which are Conway reducible.

On the other hand, if the three-component link is chosen to be $\pi$-hyperbolic, the resulting pairs of non-equivalent hyperbolic knots are Conway irreducible (in fact $\pi$-hyperbolic). In Section 2 we shall describe a standard method for constructing a $\pi$-hyperbolic link once a hyperbolic knot which admits a 2-periodic symmetry with trivial quotient is given. Thus, producing $\pi$-hyperbolic links is fairly easy, though there is no standard way to assure that they meet some extra requirements.

For this reason, although it is reasonable to think that for each fixed pair of coprime integers $n, m$ there exists infinitely many pairs of non-equivalent $\pi$-hyperbolic knots with the same $n$-fold and $m$-fold cyclic branched covers, we are only able to exhibit one single pair.

In what follows, we shall review in more detail Zimmermann's method and discuss how it must be modified to obtain examples of non-equivalent Conway irreducible hyperbolic knots with two common cyclic branched covers, before constructing explicit examples (Section 2). In Section 3 we shall consider the case where one of the covers is the double one. We shall then show that all the pairs of non-equivalent $\pi$-hyperbolic knots satisfying the conclusion of Theorem 1 for $m=2$ and $n>2$ odd are obtained via Zimmermann's construction. The last section will be devoted to the proof of Theorem 2. 
Acknowledgement. The author is grateful to the referee for suggesting improvements to the form of the paper. She is also indebted to the referee of an initial version of this paper for drawing her attention to the book of Du Val and suggesting a much simpler proof of the first part of Proposition 1.

\section{Proof of Theorem 1}

In this section we shall show how to construct, for each pair of fixed coprime integers $n, m>2$, two non equivalent Conway irreducible hyperbolic knots with the same $n$-fold and $m$-fold cyclic branched covers. Recall that a knot is Conway irreducible if it does not admit any Conway sphere, i.e. a sphere meeting the knot in four points and such that the 4-punctured sphere obtained by removing the knot is incompressible and $\partial$-incompressible in the complement of the knot. Note that a Conway sphere for a knot lifts to an incompressible torus of its 2 -fold branched cover.

Since the construction will follow closely the method described in [15], we shall start by briefly recalling Zimmermann's construction.

Assume that $L$ is a three-component link satisfying [15, Property 5]:

Property (*). L is hyperbolic, each two-component sublink of $L$ is a Hopf link (in particular, each component of $L$ is trivial) and $L$ admits a symmetry which induces a cyclic permutation of its three components but no symmetry which exchanges two components while fixing setwise the third one.

Here and in the rest of the paper a symmetry denotes a finite order diffeomorphism of the pair $\left(\mathbb{S}^{3}, L\right)$ which preserves the orientation of the 3 -sphere, but not necessarily that of the components of $L$.

We shall explain how one can use $L$ to construct two knots with two common cyclic branched covers. Let $n, m \geq 2$ be two coprime integers.

Take the cover of $\mathbb{S}^{3}$ ramified of order $n$ along one component of $L$, say $L_{1}$. Since every component of $L$ is trivial, the resulting manifold is again $\mathbb{S}^{3}$. Moreover, since each two-component sublink of $L$ is a Hopf link, the preimages $\tilde{L}_{2}$ and $\tilde{L}_{3}$, on the cover, of the remaining two components are two trivial knots whose linking number is $n$ (up to sign). Take now the cover of $\mathbb{S}^{3}$ ramified of order $m$ along the lift $\tilde{L}_{i}$ of one of the remaining components. This cover is again $\mathbb{S}^{3}$ and, since the order of the cover and the linking number of $\tilde{L}_{2}$ and $\tilde{L}_{3}$ are coprime, the lift of $\tilde{L}_{j}, i \neq j$, is connected, i.e. a knot $K$.

Remark that this construction corresponds to taking the $\mathbb{Z}_{n} \oplus \mathbb{Z}_{m} \cong \mathbb{Z}_{n m}$-cover of $\mathbb{S}^{3}$ ramified of order $n$ along one component and of order $m$ along another component of $L$. Note moreover that, since $L$ is symmetric, there are only two ways to take such cover, resulting in two -possibly equivalent- knots $K$ and $K^{\prime}$ in $\mathbb{S}^{3}$. 
By construction, $K$ and $K^{\prime}$ have the same $n$-fold and $m$-fold cyclic branched covers. Indeed, the $n$-fold cyclic branched cover (respectively $m$-fold cyclic branched cover) of $K$ and $K^{\prime}$ is the $\mathbb{Z}_{n} \oplus \mathbb{Z}_{n} \oplus \mathbb{Z}_{m}$-cover (respectively $\mathbb{Z}_{m} \oplus \mathbb{Z}_{m} \oplus \mathbb{Z}_{n}$-cover) of $\mathbb{S}^{3}$ ramified of order $n, n$ and $m$ (respectively $m, m$ and $n$ ) along the three components of $L$. Note that these covers are unique because of the symmetry of $L$. Remark that these covers can also be seen in the following way: take the $m$-fold (respectively $n$-fold) cyclic cover of $\mathbb{S}^{3}$ branched along one component of $L$ and take the lift -say $\hat{L}_{m}$ (respectively $\hat{L}_{n}$ )- of the remaining two components. The $n$-fold (respectively $m$-fold) cyclic branched cover of $K$ and $K^{\prime}$ is the $\mathbb{Z}_{n} \oplus \mathbb{Z}_{n}$ (respectively $\mathbb{Z}_{m} \oplus \mathbb{Z}_{m}$ ) cover of $\mathbb{S}^{3}$ branched along the two components of $\hat{L}_{m}$ (respectively $\hat{L}_{n}$ ).

Assume now that $K$ and $K^{\prime}$ are hyperbolic. If they were equivalent, the map $\left(\mathbb{S}^{3}, K\right) \longrightarrow\left(\mathbb{S}^{3}, K^{\prime}\right)$ would induce a symmetry of $\hat{L}_{m}$ and of $\hat{L}_{n}$ exchanging the two components and a symmetry of $L$ exchanging two of its components while fixing setwise the third one (see [15] and [16, Theorem 1] for more details); the converse is obvious.

We shall need the following version of Thurston's orbifold geometrization theorem (see [1], [4] for a proof).

Theorem (Thurston). A link $L=L_{1} \cup \ldots \cup L_{k}$ is hyperbolic if it is $\left(2 \pi / n_{1}, \ldots\right.$, $\left.2 \pi / n_{k}\right)$-hyperbolic for some choice of $n_{i}>1, i=1, \ldots, k$, and in this case it is also $\left(2 \pi / n_{1}^{\prime}, \ldots, 2 \pi / n_{k}^{\prime}\right)$-hyperbolic for all choices of $n_{i}^{\prime}, i=1, \ldots, k$, such that $n_{i}^{\prime} \geq n_{i}$. Moreover, a hyperbolic link is $2 \pi / 3$-hyperbolic provided it is not the figure-eight knot $4_{1}$ in which case it is $\pi / 2$-hyperbolic.

Recall that a link $L=L_{1} \cup \cdots \cup L_{k}$ in $\mathbb{S}^{3}$ is said to be $\left(2 \pi / n_{1}, \ldots, 2 \pi / n_{k}\right)$ hyperbolic if the orbifold with underlying topological space $\mathbb{S}^{3}$ and singular set $L$, where the order of singularity of $L_{i}$ is $n_{i}, i=1, \ldots, k$, is hyperbolic. If $n_{i}=n$ for all $i=1, \ldots, k, L$ is simply called $2 \pi / n$-hyperbolic.

Suppose that $L$ is a $\pi$-hyperbolic three-component link. Then, by Thurston's orbifold geometrization theorem, $L$ is $(2 \pi / n, 2 \pi / m, \pi)$-hyperbolic and also $(2 \pi / m, 2 \pi / n, \pi)$-hyperbolic, and it follows from their construction that the knots $K$ and $K^{\prime}$ are $\pi$-hyperbolic.

To construct the desired examples it is then sufficient to find a $\pi$-hyperbolic link satisfying Property $(*)$ given above. Note that to build a $\pi$-hyperbolic link, one can use a hyperbolic knot admitting a 2-periodic symmetry in the following way. Let $\bar{K} \cup \bar{A}$ be the image of the knot and of the axis of symmetry respectively in the quotient of the 3 -sphere via the action of the symmetry, and assume that $\bar{K}$ is the trivial knot. Consider now the $n$-fold cyclic cover of $\mathbb{S}^{3}$ branched over $\bar{K}$ : this is again $\mathbb{S}^{3}$ and, if $K$ is $2 \pi / n$-hyperbolic, the lift of $\bar{A}$ is a $\pi$-hyperbolic link. In the following we shall choose an appropriate hyperbolic knot for which the aforementioned construction gives a link satisfying the remaining requirements of Property $(*)$. 
Let $\mathcal{K}$ be the knot $10_{147}$ (according to Rolfsen's notation [12]): it is a non elliptic Montesinos knot, thus it is hyperbolic and $2 \pi / 3$-hyperbolic, and admits a unique symmetry $\rho$ which is a 2-periodic symmetry (see [2]). Take the quotient of $\mathbb{S}^{3}$ by the action of the symmetry $\rho$. The link $\overline{\mathcal{K}} \cup \bar{A}$ which is the image in such quotient of $\mathcal{K}$ and the axis $A$ of $\rho$ is a two component Montesinos link, which admits a unique symmetry of order 2 , exchanging its components. Note that, by construction, this link is $(2 \pi / 3, \pi)$-hyperbolic and since its two components are exchangeable it is also $(\pi, 2 \pi / 3)$-hyperbolic. The 3 -fold cyclic covering of $\mathbb{S}^{3}$ branched along $\bar{A}$ is again $\mathbb{S}^{3}$ and the preimage of $\overline{\mathcal{K}}$ is a three-component $\pi$-hyperbolic link $L$.

Figure 1 shows the three links $\mathcal{K}, \overline{\mathcal{K}} \cup \bar{A}$ and $L ; \overline{\mathcal{K}} \cup \bar{A}$ is represented as the quotient of $\mathcal{K}$ and as the closure of a 5-tangle around an axis. In Figure 2 we show

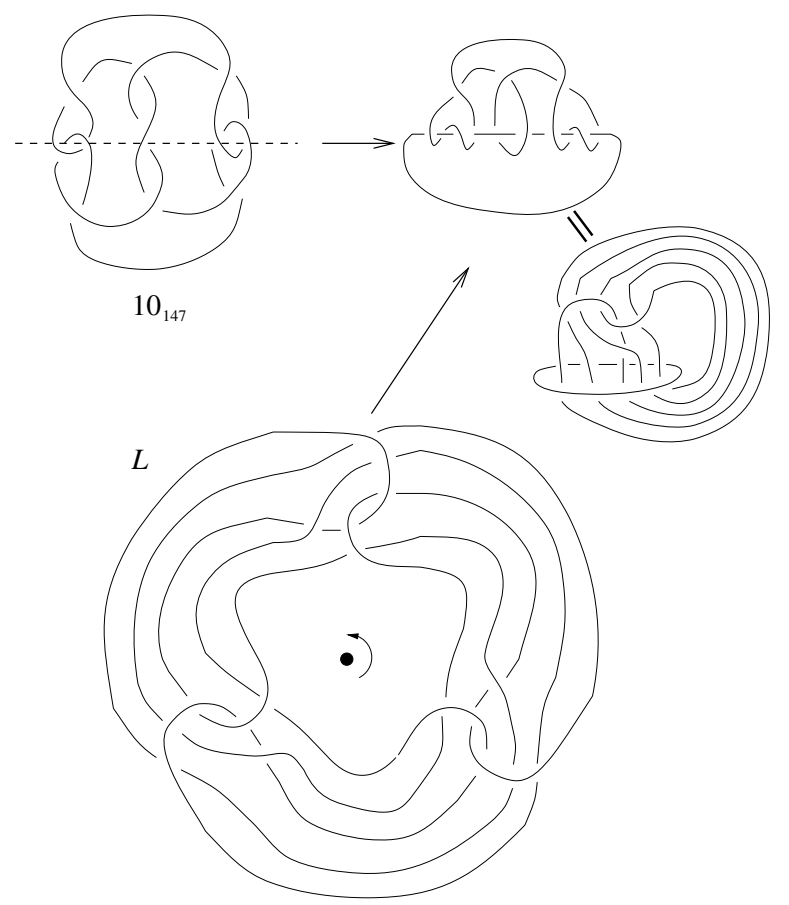

Figure 1

how one can pass via isotopy from the first diagram of $\overline{\mathcal{K}} \cup \bar{A}$ (i.e. the quotient of $\mathcal{K}$ ) to the second diagram (i.e. the quotient of $L$ ): the 5 -tangle can be seen by cutting $\mathbb{S}^{3}$ open along the shaded disc bounded by the axis and pulling up the strands passing behind.

Proposition 1. The link $L$ verifies Property (*) and its symmetry group is $\mathbb{Z}_{3}$. 


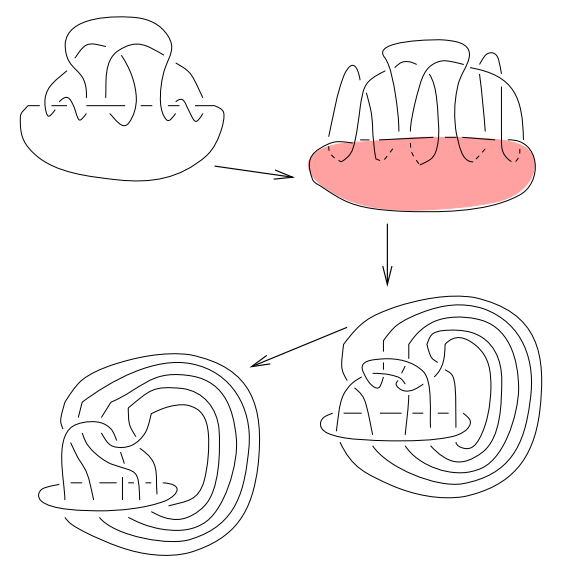

Figure 2

Proof. To see that each two-component sublink of $L$ is a Hopf link it suffices to look at the picture. By construction, $L$ admits a symmetry of order $3, \sigma$, which cyclically exchanges its components. We thus have to show that $L$ does not admit symmetries which exchange two components and fix the remaining one. Let $G$ be the group of symmetries of $L$, then the normalizer of $\langle\sigma\rangle$ in $G$ coincides with $\langle\sigma\rangle$. This follows from the fact that the elements of $\mathcal{N}_{G}\langle\sigma\rangle /\langle\sigma\rangle$ induce non trivial symmetries of $\overline{\mathcal{K}} \cup \bar{A}$ which fix setwise both its components. However, the only non trivial symmetry of $\overline{\mathcal{K}} \cup \bar{A}$ is an involution which exchanges its components [2]. Note, moreover, that there is a homomorphism from $G$ to the symmetric group on three elements, $S_{3}$, defined by the permutation induced by the elements of $G$ on the three components of $L$. To end the proof of the first part of the proposition it is then sufficient to show that this homomorphism is not surjective.

Since $\sigma$ has non-empty fixed-point set, Thurston's orbifold geometrization theorem implies that $G$ is linear, i.e. a finite subgroup of $\mathrm{SO}(4)$, moreover it does not contain the central involution. The list of all such groups can be found in [5, page 57] and a case by case check shows that elements of order 3 always have non trivial normalizers unless the group is either cyclic of order $3, \mathbb{Z}_{3}$, or alternating on 4 elements, $\mathrm{A}_{4}$. However, these two groups do not surject onto $\mathrm{S}_{3}$.

Now we only need to prove that $G$ cannot be $\mathrm{A}_{4}$. Indeed, all three involutions of $\mathrm{A}_{4}$ are conjugate and must fix setwise each component of $L$. Since each two-component sublink of $L$ is the Hopf link, each symmetry fixing setwise the components of $L$ must either preserve or reverse the orientation of each component and since the involutions are all conjugate and each one of them is the product of the remaining two, they all must preserve the orientations of the components of $L$. There are two cases to be considered: either the fixed-point set of each involution is non-empty and coincides 
with one component of $L$ (a different component for each involution) or they all act as non trivial translations along each component of $L$. The latter case is not possible since the product of two order 2 translations would be the identity, against the hypothesis. We can thus assume to be in the former case. Let $\eta$ be the involution fixing pointwise the first component $L_{1}$ of $L$. Consider $\eta$ as a symmetry of the trivial knot $L_{2}$. Using [6, Theorem 5.2] we find a fibration by discs of the solid torus $\mathbb{S}^{3} \backslash L_{2}$ such that each fibre is invariant by the action of $\eta$. Observe now that $L_{3}$ is also invariant by the action of $\eta$ but this is impossible since the linking number of $L_{2}$ and $L_{3}$ is 1 (note that $\operatorname{lk}\left(L_{2}, L_{3}\right)$ is the algebraic intersection of each disc of the fibration with $\left.L_{3}\right)$.

Using Week's program SnapPea, one can check directly that $G \cong \mathbb{Z}_{3}$.

\section{Double covers}

We have seen that, for each fixed odd $n>2$, it is possible to produce pairs of non equivalent $\pi$-hyperbolic knots with the same 2 -fold and $n$-fold cyclic branched covers. In this section we wish to show that all such knots, which constitute counterexamples to the main result of [8], can be obtained via Zimmermann's construction, as the following result shows:

Proposition 2. Let $n>2$ be an odd integer and $K$ and $K^{\prime}$ two non equivalent $\pi$ hyperbolic knots having the same 2-fold and $n$-fold cyclic branched covers. Up to conjugation, the covering involutions for $K$ and $K^{\prime}$, acting on their common 2-fold branched cover, commute and have disjoint axes. In particular $K$ admits a 2- and an n-periodic symmetry with disjoint axes $A, B$. The quotient of $\left(\mathbb{S}^{3}, K \cup A \cup B\right)$ by the action of these symmetries gives a three component link satisfying Property (*).

Proof. In [16] Zimmermann proved that if a hyperbolic knot is not determined by its $n$-fold cyclic branched cover, then it must admit an $n$-periodic symmetry with trivial quotient, thus both $K$ and $K^{\prime}$ admit $n$-periodic symmetries, moreover there exists a link with two trivial components $\bar{K} \cup \bar{K}^{\prime}$ such that $K$ (respectively $K^{\prime}$ ) is the lift of $\bar{K}$ (respectively $\bar{K}^{\prime}$ ) to the $n$-fold cyclic cover of $\mathbb{S}^{3}$ branched along $\bar{K}^{\prime}$ (respectively $\bar{K})$. Let $M$ be the hyperbolic manifold which is the common 2-fold branched cover of $K$ and $K^{\prime}$. Consider the following isometries of $M: \tau$ respectively $\tau^{\prime}$ are covering involutions for $K$ respectively $K^{\prime}$, while $h$ respectively $h^{\prime}$ are lifts of their $n$-periodic symmetries. Note that $\tau$ and $h$, respectively $\tau^{\prime}$ and $h^{\prime}$ commute. It was proved in [10, Claim 2(e)], that $h$ and $h^{\prime}$ are conjugate in the isometry group of $M$. Thus, up to conjugation, the isometries $h=h^{\prime}, \tau$ and $\tau^{\prime}$ generate a group isomorphic to $\mathbb{Z}_{n} \oplus \mathrm{D}_{2 d}$, where $\mathrm{D}_{2 d}$ denotes the dihedral group of order $4 d$ generated by $\tau$ and $\tau^{\prime}$. Remark that its maximal cyclic group cannot have odd order, else $\tau$ and $\tau^{\prime}$ would be 
conjugate, against the hypothesis that $K$ and $K^{\prime}$ are non equivalent. Let $r=\left(\tau \tau^{\prime}\right)^{d}$ be the central involution. Consider now the involution $r \tau$, which commutes with $\tau$. A straightforward computation shows that this involution is conjugate to $\tau$, if $d$ is even, or to $\tau^{\prime}$, if $d$ is odd. Note that the fixed-point set of $r \tau$ cannot meet that of $\tau$ since $r \tau$ and $h$ commute, in other words $r \tau$ cannot be a strong inversion for strong inversions conjugate periodic symmetries to their inverses. If $d$ is odd, we can then choose $\tau^{\prime}=r \tau$ and the assertion follows.

We can thus assume that $d$ is even and that $r \tau$ (respectively $r \tau^{\prime}$ ) is conjugate to $\tau$ (respectively $\tau^{\prime}$ ) and commutes with $\tau$ (respectively $\tau^{\prime}$ ) and $h$. This means that both $r \tau$ and $r \tau^{\prime}$ induce involutions of the hyperbolic link $\bar{K} \cup \bar{K}^{\prime}$ with non-empty fixed-point set and acting as 2-periodic symmetries on both components, in particular the two involutions they induce must coincide. Indeed, if this were not the case, the product of the two symmetries would fix pointwise a two-component link against the fact that fixed-point sets of diffeomorphisms of prime order acting on a homology sphere must be connected according to Smith's fixed-point theory.

Call $\phi$ the involution thus obtained. Let $L_{1} \cup L_{2} \cup L_{3}$ denote the three component link which is the image of $\bar{K} \cup \bar{K}^{\prime}$ and $\operatorname{Fix}(\phi)$ in the quotient of $\mathbb{S}^{3}$ via the action of $\phi$. Let $\hat{K} \cup \hat{A}$ be the images of $K$ and the axis of the 2-periodic symmetry of $K$ induced by $r \tau$ in the quotient of $\mathbb{S}^{3}$ via the action of the 2-periodic symmetry. Define in a similar way $\hat{K}^{\prime} \cup \hat{A}^{\prime}$ for $K^{\prime}$ and $r \tau^{\prime}$.

Note now that, since $r \tau$ is conjugate to $\tau$, the two components of $\hat{K} \cup \hat{A}$ are exchangeable and project to $L_{1} \cup L_{3}$. Analogously, the two components of $\hat{K}^{\prime} \cup \hat{A}^{\prime}$ are exchangeable and project to $L_{2} \cup L_{3}$. This means that the components of $L_{1} \cup L_{2} \cup L_{3}$ are exchangeable, too, and $K$ and $K^{\prime}$ are equivalent which is against the hypotheses.

Let $L=L_{1} \cup L_{2} \cup L_{3}$ the link obtained by quotienting $K$ via its 2-periodic and $n$-periodic symmetries. Any easy check (compare [10, §3.1]) shows that its three components must be cyclically exchangeable. To see that each two-component sublink of $L$ is a Hopf link one uses the fact that, given a periodic symmetry of a trivial knot, the knot together with the axis of the symmetry form a Hopf link (see [10, Claim 1]).

The situation described by Proposition 2 is very special because the covering involutions acting on a common double branched cover of two non-equivalent hyperbolic knots need not commute up to conjugation (see [7]) and, even if they commute, their fixed-point sets may intersect (see $[15, \S 5])$.

\section{Proof of Theorem 2}

First of all note that Theorem 2 is true if the knot $K$ is Conway irreducible but not $\pi$-hyperbolic [8, proof of Proposition 1] or if it is $\pi$-hyperbolic but $n$ is even 
[16, Theorem 3] even without assuming that $K$ admits a unique incompressible Seifert surface up to isotopy.

For $n$ odd and $K \pi$-hyperbolic the proof is the same as that of [8, Theorem 2]. Some care is needed to apply $[8, \mathrm{Lemma}]$. In fact, in the proof of $[8, \mathrm{Lemma}]$ it is incorrectly assumed that, one can choose a minimum genus Seifert surface which is equivariant by a cyclic group $G$ of symmetries preserving the orientation of the knot in such a way that the quotients of the surface via the action of two distinct subgroups of periodic symmetries of $G$ are again minimum genus Seifert surfaces for the quotient knots. This is nevertheless the case if the knot admits a unique Seifert surface up to isotopy:

Lemma 1. Let $h$ and $g$ be two commuting periodic symmetries of a knot $K$ which generate distinct subgroups and let $K_{h}$ (respectively $K_{g}$ ) be the quotient of $K$ by the action of $h$ (respectively $g$ ). If $K$ admits a unique Seifert surface up to isotopy, it is possible to find a minimum genus Seifert surface for $K$ which is $\langle h\rangle$ - and $\langle g\rangle$-equivariant and such that the quotients of the surface by the action of $h$ and of $g$ are again minimum genus Seifert surfaces for $K_{h}$ and $K_{g}$ respectively.

Proof. Let $\mathscr{H}$ (respectively $g$ ) be a minimum genus Seifert surface for $K_{h}$ (respectively $K_{g}$ ) which is equivariant by the action induced by $g$ (respectively $h$ ). The existence of such surfaces is proved in [13, Theorem 6]. Let $F$ and $F^{\prime}$ be the equivariant Seifert surfaces for $K$ obtained as lifts of $\mathcal{H}$ and $g$. According to the equivariant loop theorem-Dehn lemma $F$ and $F^{\prime}$ are incompressible and, because of the hypothesis, are isotopic and have minimum genus.

It is now sufficient to use the fact, which was pointed out to the author by M. Boileau, that two isotopic equivariant incompressible Seifert surfaces are equivariantly isotopic. We shall propose here the proof of this fact which follows the lines of [3, Proposition 4.5] where the case of $\mathbb{Z}_{2}$-actions is considered.

Lemma 2. Let $F$ and $F^{\prime}$ be two incompressible isotopic Seifert surfaces for a knot $K$ which are invariant by the action of a finite cyclic group $G$. Assume that the elements of $G$ preserve the orientation of $\mathbb{S}^{3}$ and of $K$. Then there exists an equivariant isotopy between $F$ and $F^{\prime}$.

Proof. By a general position argument we can assume that $F$ and $F^{\prime}$ intersect transversally only in a finite number of simple closed curves. Notice that we can assume that $\partial F=\partial F^{\prime}=K$. We shall now reason by induction on the number of connected components in $F \cap F^{\prime}$ and apply several times the following result due to Waldhausen [14, Proposition 5.4]:

Proposition (Waldhausen). Let $F$ and $F^{\prime}$ be two incompressible surfaces contained in an irreducible manifold $M$ such that $\partial F=\partial F^{\prime}$ and such that their intersection 
consists of mutually disjoint simple closed curves with transversal intersection outside $\partial F=\partial F^{\prime}$. Assume that $F$ and $F^{\prime}$ are isotopic. Then there exists a surface $\&$ and an embedding $\delta \times I \longrightarrow M$, such that $\delta \times 0=S \subset F, \partial(\delta \times I) \backslash \delta \times 0=S^{\prime} \subset F^{\prime}$ and that moreover $S \cap F^{\prime}=\partial S$, and either $S^{\prime} \cap F=\partial S^{\prime}$ or $S$ and $S^{\prime}$ are discs.

If $F \cap F^{\prime}=\partial F=\partial F^{\prime}$, then, by the above proposition, there is a product region between $F$ and $F^{\prime}$, i.e. an embedding of $F \times[0,1]$ such that the image of $F \times\{0\}$ is $F$, that of $F \times\{1\} \cup \partial F \times[0,1]$ is $F^{\prime}$. It is easy to see that the product is invariant by the action of $G$, since the elements of $G$ preserve the orientation of the sphere and of the Seifert surfaces. Using [6, Theorem 4.1], we see that the action of $G$ preserves the product structure and we are done.

If the intersection consists of more than one component, again by [14, Proposition 5.4], we can find a product region between two subsurfaces of $F$ and $F^{\prime}$. Take an innermost product region $P$, i.e. one that does not contain any other product region, between two subsurfaces $S$ and $S^{\prime}$ of $F$ and $F^{\prime}$ respectively. According to the above proposition, two situations can arise. Assume first that $S \cap F^{\prime}=\partial S$ and $S^{\prime} \cap F=\partial S^{\prime}$. In this case, since the elements of $G$ preserve the orientations of $F$ and $F^{\prime}$ and preserve $F \cap F^{\prime}$, we have that the $G$-orbit of $P$ consists of pairwise disjoint product regions (i.e. two translates are either disjoint or coincide). In particular, the above argument applies to reduce equivariantly the intersection.

We can thus assume that both $S$ and $S^{\prime}$ are discs and that, without loss of generality, $S^{\prime}$ intersects $F$ outside $\partial S^{\prime}$, while $S \cap F^{\prime}=\partial S$, i.e. $S$ is innermost. In particular $P$ is a ball whose interior does not intersect $F^{\prime}$. Since $S$ is an innermost disc, the elements of $G$ either fix $S$ setwise or send it to another innermost disc disjoint from $S$. Consider a connected component $C$ of $F \cap P$, contained in the interior of $P$. We want to show that $C$ cannot be a disc. If $C$ were a disc, $\partial C$ would bound a disc $\Delta$ on $S^{\prime}$. $C$ and $\Delta$ would then bound a ball inside $P$, against the hypothesis that $P$ is an innermost product region. In particular, the $G$-orbit of $S$ intersects $P$ only in $S$. This implies that the $G$-orbit of $P$ consists of pairwise disjoint balls. Since $P$ does not meet $F^{\prime}$ in its interior, $S^{\prime}$ can be equivariantly isotoped onto $S$ in such a way as to diminish the number of curves in $F \cap F^{\prime}$.

\section{References}

[1] M. Boileau, J. Porti, Geometrization of 3-orbifolds of cyclic type. Appendix A by M. Heusener and J. Porti. Astérisque 272, Soc. Math. France, Paris 2001. Zbl 0971.57004 MR 1844891

[2] M. Boileau, B. Zimmermann, Symmetries of nonelliptic Montesinos links. Math. Ann. 277 (1987), 563-584. Zbl 0608.57010 MR 0891592

[3] M. Boileau, B. Zimmermann, The $\pi$-orbifold group of a link. Math. Z. 200 (1989), 187-208. Zbl 0663.57006 MR 0978294 
[4] D. Cooper, C. D. Hodgson, S. P. Kerckhoff, Three-dimensional orbifolds and conemanifolds. With a postface by S. Kojima. MSJ Memoirs 5, Mathematical Society of Japan, Tokyo 2000. Zbl 0955.57014 MR 1778789

[5] P. Du Val, Homographies, quaternions and rotations. Oxford Mathematical Monographs, Clarendon Press, Oxford 1964. Zbl 0128.15403 MR 0169108

[6] A. L. Edmonds, C. Livingston, Group actions on fibered three-manifolds. Comment. Math. Helv. 58 (1983), 529-542. Zbl 0532.57024 MR 0728451

[7] M. Mecchia, Hyperbolic 2-fold branched coverings. Rend. Istit. Mat. Univ. Trieste 32, suppl. 1, (2001), 165-180. Zbl 1009.57008 MR 1893397

[8] L. Paoluzzi, On $\pi$-hyperbolic knots and branched coverings. Comment. Math. Helv. 74 (1999), 467-475. Zbl 0942.57003 MR 1710147

[9] L. Paoluzzi, Non-equivalent hyperbolic knots. Topology Appl. 124 (2002), 85-101. Zbl 1024.57006 MR 1926137

[10] L. Paoluzzi, Three cyclic branched covers suffice to determine hyperbolic knots. To appear in J. Knot Theory Ramifications.

[11] L. Paoluzzi, Erratum to "On $\pi$-hyperbolic knots and branched coverings". Comment. Math. Helv. 80 (2005), 225-227.

[12] D. Rolfsen, Knots and links. Publish or Perish, Berkeley 1976. Zbl 0339.55004 MR 0515288

[13] J. L. Tollefson, Innermost disk pairs in least weight normal surfaces. Topology Appl. 65 (1995), 139-154. Zbl 0845.57013 MR 1355291

[14] F. Waldhausen, On irreducible 3-manifolds which are sufficiently large. Ann. Math. (2) 87 (1968), 56-88. Zbl 0157.30603 MR 0224099

[15] B. Zimmermann, On hyperbolic knots with the same $m$-fold and $n$-fold cyclic branched coverings. Topology Appl. 79 (1997), 143-157. Zbl 0888.57006 MR 1464193

[16] B. Zimmermann, On hyperbolic knots with homeomorphic cyclic branched coverings. Math. Ann. 311 (1998), 665-673. Zbl 0913.57008 MR 1637960

Received October 5, 2004

Luisa Paoluzzi, IMB - UMR 5584 du CNRS, Université de Bourgogne, avenue Alain Savary, BP 47870, 21078 Dijon Cedex, France

E-mail: paoluzzi@u-bourgogne.fr 